\title{
Alternative tumor markers in the diagnosis of ovarian cancer
}

\author{
Burcu Kısa Karakaya ${ }^{1}$, Eralp Başer ${ }^{1}$, Berk Bildacı ${ }^{1}$, Esra Çabuk Cömert ${ }^{1}$, Nilufer Bayraktar², \\ Polat Dursunn ${ }^{1}$, Esra Kuşçu ${ }^{1}$, Ali Ayhan ${ }^{1}$ \\ ${ }^{1}$ Department of Obstetrics and Gynecology, Başkent University School of Medicine, Ankara, Turkey \\ ${ }^{2}$ Department of Biochemistry, Başkent University School of Medicine, Ankara, Turkey
}

\begin{abstract}
Objectives: The aim of the study was to assess the usefulness of various tumor markers (CA125, HE4, bcl2) measured in serum, urine and saliva in the differential diagnosis of adnexal masses.

Material and methods: Our study was conducted at the Başkent University Medical School, Department of Obstetrics and Gynecology, Ankara, Turkey, between November 2010 and March 2011. Fifty patients with a suspicion of malignant adnexal mass and 30 controls were included in the study. Serum and urine CA-125, HE4, and bcl2 levels were evaluated for their role in the diagnosis of epithelial ovarian cancer (EOC).

Results: Serum CA-125 and HE4 levels, and urine HE4 levels were significantly higher in malignant cases as compared to controls $(p<0.05)$. Mean levels of $b c l 2$ in saliva and urine were similar in malignant cases and controls $(p>0.05)$.

Conclusions: We demonstrated that serum CA125, serum HE4 and urine HE4 levels were elevated in patients with ovarian cancer. These findings should be assessed in future studies with larger sample sizes in order to reach more definite conclusions.
\end{abstract}

Key words: adnexal mass, ovarian cancer, tumor marker, CA125, HE4, bcl2

Ginekologia Polska 2016; 87, 8: 565-569

\section{INTRODUCTION}

Annually, 204.000 women are diagnosed with ovarian cancer (OC), with 125.000 dying of the disease [1]. According to Globocan 2008, 1804 patients were diagnosed with ovarian cancer and 1204 died of the disease [2]. In the absence of effective screening methods, approximately $70 \%$ of OC cases are diagnosed at advanced stages of the disease [3]. The 5-year overall survival following the diagnosis at an early stage is nearly $90 \%$, which is in contrast with the $30 \%$ survival following diagnosis at an advanced stage [3]. In addition, the 5-year recurrence rate for patients diagnosed at advanced stages is about $80 \%$ [4]. These data underscore the importance of early diagnosis in this potentially lethal disease.

The established diagnostic utilities in ovarian cancer include pelvic examination, transvaginal ultrasound (TVUS), and serum tumor markers. With annual pelvic examina- tions, early-stage $O C$ is detected in $1 / 10.000$ women [5]. Currently, CA125 remains to be the most commonly used tumor marker. CA125 is a glycoprotein marker which is elevated in $50 \%$ of early and $80-90 \%$ of advanced stages of the disease [6]. Moreover, CA125 levels may increase in benign gynecologic and non-gynecologic conditions as well. When used alone, OC may be detected in 1 out of 1000 screened subjects. Thus, the CA125 measurement alone is inadequate as a screening tool [5]. TVUS is considered to be the most effective imaging method to visualize the ovaries. The sensitivity of this method for diagnosing ovarian malignancies has been estimated at 48-92\% [7]. Various combinations of these diagnostic modalities have been tested to define the optimal method.

Novel tumor markers are currently being investigated for the early diagnosis of OC. Among these, HE4 and bcl2 seem most promising. However, more data on the 
effectiveness and feasibility of these markers are needed to incorporate them into routine clinical practice. Besides serum, body fluids which can be acquired in a non-invasive fashion, e.g. urine and saliva, are also being investigated for their potential to be tested for tumor markers [8-11]. Human epididymis protein-4 (HE4) and bcl2 (an anti-apoptotic marker) have been demonstrated to be elevated in urine of OC patients $[8,9] . \mathrm{Bcl} 2$ is secreted into all body fluids and has been proposed as a novel tumor marker in early-stage OC $[8,12]$.

\section{OBJECTIVES}

In the present study, the utility of CA125, HE4 and bcl2 tumor markers was investigated for ovarian cancer diagnosis in patients with suspicious adnexal masses.

\section{MATERIAL AND METHODS}

This cross-sectional study was conducted at the Department of Gynecologic Oncology, Başkent University Medical School, Ankara, Turkey. Local Ethics Committee approved of the study (KA10/62). Fifty patients, diagnosed with a suspicious adnexal mass between October 2010 and March 2011, constituted the study group, and 30 healthy non-pregnant women who presented to our institution for a regular gynecologic examination comprised the control group.

Serum, urine, and saliva samples were preoperatively obtained from the study group, and at the time of the clinical examinations after gathering the informed consent from the control group. Serum was tested for CA125 and HE4 levels. Blood samples were centrifuged at $10800 \mathrm{rpm}$ for $10 \mathrm{~min}$, and the collected serum was stored at $-80^{\circ} \mathrm{C}$. Urine samples were tested for HE4 and bcl2 levels. Urine samples were centrifuged at $3500 \mathrm{rpm}$ for $15 \mathrm{~min}$, and the supernatant fluid was stored at $-80^{\circ} \mathrm{C}$. Saliva samples were obtained from all patients using Diametra Saliva Collection Device tubes and pipettes, and were kept at $-20^{\circ} \mathrm{C}$ for 24 hours. Next, the saliva samples were thawed, mixed, and homogenized using a vortex. The samples were centrifuged at $3500 \mathrm{rpm}$ for $15 \mathrm{~min}$, and the supernatant fluid was again collected and frozen at $-20^{\circ} \mathrm{C}$. This procedure was performed to increase the viscosity of the saliva samples.

Serum and urine HE4 levels were measured using the enzyme immunometric assay (EIA) method (Fujirebio Diagnostics Inc., Sweden). This method is a solid phase non-competitive measurement, which is dependent on direct sandwich technique using 2H5 and 3D8 monoclonal antibodies directed against two epitopes on the HE4 C-WFDC chain. Picomolar (pM) units were utilized for HE4. Urine HE4 levels were assessed by measuring urine creatinine, and calculating a normalized HE4 (pM)/urine creatinine $(\mathrm{mg} / \mathrm{dL})$ ratio.
The Risk of Malignancy Algorithm (ROMA) is a serum biomarker algorithm that aims to determine the risk of $\mathrm{OC}$ in women with an adnexal mass, and combines CA125, $\mathrm{HE}$, and the menopausal status. The formulas used in this calculation were as follows:

(1) Premenopausal women

Predictive index

$(\mathrm{PI})=-12.0+2.38^{*} \mathrm{LN}[\mathrm{HE} 4]+0.0626^{*} \mathrm{LN}[\mathrm{CA} 125]$

(2) Postmenopausal women

Predictive index

$(\mathrm{PI})=-8.09+1.04 * \mathrm{LN}[\mathrm{HE} 4]+0.732 * \mathrm{LN}[\mathrm{CA} 125]$

\section{Calculation of ROMA values}

(3) ROMA value percentage $\%=\exp (\mathrm{PI}) /[1+\exp (\mathrm{PI})] \times 100$ Premenopausal $>12.5$ postmenopausal $>14.4$ high risk for epithelial ovarian cancer

Premenopausal $<12.5$ postmenopausal $<14.4$ low risk for epithelial ovarian cancer

Urine and saliva bcl2 levels ( $\mathrm{ng} / \mathrm{mL}$ ) were assessed using the enzyme-linked immunosorbent assay — ELISA (Biovendor LM Inc; Czech Republic), with the sensitivity of $<0.5 \mathrm{ng} / \mathrm{mL}$. Serum CA125 levels $(\mathrm{U} / \mathrm{mL})$ were measured using chemiluminescence microparticle immunoassay (CMIA) (Abbott Architect i2000; Abbott Laboratories; IL, USA). Patient charts were retrieved from the hospital computer archives. Clinical data were evaluated, together with CA125, HE4, bcl2 and the final pathology reports.

\section{Statistical analysis}

Study data were analyzed with SPSS 17.0 computer software (Statistical Package for Social Sciences, SPSS Inc, Chicago, IL, USA). Categorical variables were expressed as count and percentage, whereas continuous variables were expressed as mean \pm standard deviation (SD). Chi-square, Kruskal-Wallis and one-way ANOVA tests were used to compare the study groups and subgroups, where appropriate. P-values of $<0.05$ were considered as statistically significant.

\section{RESULTS}

A total of 80 subjects were included in the study. Fifty patients with an adnexal mass comprised the study group, and 30 healthy women were recruited as controls. Sociodemographic and clinical characteristics of the study population are presented in Table 1. Out of all patients with an adnexal mass, 32 were diagnosed with OC or borderline ovarian tumor (BOT) on the final pathology examination, and 18 were found to have benign adnexal masses. Among the neoplastic cases, 24 patients had OC, 7 had BOT, and 1 had a metastatic gastric cancer (Krukenberg tumor). Twenty-three of the 24 OC subjects had epithelial ovarian cancer (EOC), and 1 had a sex cord-stromal tumor. Of the patients with 
a benign ovarian pathology, 13 had an endometriotic cyst, 1 dermoid cyst, 1 serous cystadenoma, 1 mucinous cystadenoma, and 1 torsioned ovarian cyst. Patients with OC were significantly older than patients with a benign ovarian pathology (54.5 \pm 15.34 vs. $32.39 \pm 12.1$ years, respectively) $(p<0.001)$. The postmenopausal status was more common in malignant cases. Fourteen (56.3\%) of the malignant cases and 10 (33.3\%) of the benign cases were postmenopausal. Of the 23 patients with EOC, 18 had stage III-IV (78.3\%) disease at the time of diagnosis, whereas $5(21.7 \%)$ patients had stage I disease.

The study group (women with an adnexal mass) was further subdivided into two groups (malignant and benign).

Table 1. Sociodemographic and clinical characteristics of the study population

\begin{tabular}{|l|c|c|}
\hline & Study group (n: 50) & Control group (n: 30) \\
\hline Age & $\begin{array}{c}46.54 \pm 17.75 \\
(\min 19-\max 84)\end{array}$ & $\begin{array}{c}40.5 \pm 14.44 \\
(\min 23-\max 73)\end{array}$ \\
\hline Gravidity & $1.76 \pm 1.74$ & $1.13 \pm 0.97$ \\
\hline Parity & $1.62 \pm 1.61$ & $1.03 \pm 0.92$ \\
\hline Premenopausal & $31(62 \%)$ & $20(66.6 \%)$ \\
\hline Postmenopausal & $19(38 \%)$ & $10(33.3 \%)$ \\
\hline CA125 (U/mL) & $461.08 \pm 970.31$ & $16.08 \pm 6.86$ \\
\hline
\end{tabular}

A total of 3 groups, including controls, were then compared in terms of tumor marker levels (Table 2). Mean tumor marker levels were also compared with regard to the menopausal status (Table 3). Patients with the final pathology diagnosis of endometriosis and EOC were compared. In patients with endometriosis, mean serum CA125 was $165.63 \pm 192.91 \mathrm{U} / \mathrm{mL}$, mean serum HE4 was $70.36 \pm 43.32 \mathrm{pM}$, mean urine HE4 was $800.01 \pm 256.38 \mathrm{pM}$. In patients with EOC, mean serum CA125 was $878.65 \pm 1316.22 \mathrm{U} / \mathrm{mL}$, serum HE4 was $270.64 \pm 358.25 \mathrm{pM}$, and urine HE4 was $954.50 \pm 264.196$ pM. Mean serum CA125 and serum HE4 levels were significantly higher in patients with EOC $(p<0.05)$.

CA125 and serum HE4 levels were analyzed together according to the ROMA scoring system. Out of the 31 premenopausal patients, 19 (61.3\%) were classified as having low risk, whereas 12 (38.7\%) were grouped as high-risk. Seven patients (36.8\%) in the postmenopausal group were low-risk, and 12 (63.2\%) were high-risk, according to ROMA. The sensitivity, specificity, positive predictive values (PPV), and negative predictive values (NPV) for the ROMA score to detect malignancy in postmenopausal patients were $66.7 \%, 38.5 \%, 50 \%$, and $55.6 \%$, respectively. In premenopausal patients with ovarian malignancy, however, sensitivity, specificity, PPV and NPV values were $41.7 \%, 52.6 \%, 35.7 \%$, and $58.8 \%$, respectively.

Table 2. Comparison of tumor marker levels between women with malignant adnexal masses, benign adnexal masses, and controls

\begin{tabular}{|l|c|c|c|c|c|}
\hline Group & $\begin{array}{c}\text { CA125 } \\
\text { Median (min-max) } \\
\text { Mean } \pm \text { SD }\end{array}$ & $\begin{array}{c}\text { Serum HE4 } \\
\text { Median (min-max) } \\
\text { Mean } \pm \text { SD }\end{array}$ & $\begin{array}{c}\text { Urine HE4 } \\
\text { Median (min-max) } \\
\text { Mean } \pm \text { SD }\end{array}$ & $\begin{array}{c}\text { Urine Bcl2 } \\
\text { Median (min-max) } \\
\text { Mean } \pm \text { SD }\end{array}$ & $\begin{array}{c}\text { Saliva bcl2 } \\
\text { Median (min-max) } \\
\text { Mean } \pm \text { SD }\end{array}$ \\
\hline Malignant & $105.5(7.3-4791)$ & $61.6(7.6-1362)$ & $1010(24.95-1247)$ & $1.43(0.5-1.86)$ & $1.52(1.06-12.4)$ \\
\hline Benign & $645.18 \pm 1172$ & $202.11 \pm 321$ & $968.35 \pm 229.68$ & $1.32 \pm 0.30$ & $2.64 \pm 2.59$ \\
\hline Controls & $87(6.3-772)$ & $59.38(4.5-152.5)$ & $858.5(327-1215)$ & $1.44(0.5-1.52)$ & $1.7(1.50-10.52)$ \\
\hline $\mathbf{P}$ & $133.79 \pm 172.02$ & $66.96 \pm 41.46$ & $811.72 \pm 265.88$ & $1.34 \pm 0.25$ & $2.39 \pm 2.13$ \\
\hline & $15.9(6.8-38)$ & $84(5.6-254)$ & $799.50(295-1313)$ & $1.49(1.18-1.68)$ & $1.64(1-12.4)$ \\
\hline
\end{tabular}

Table 3. Comparison of tumor marker levels according to the menopausal status

\begin{tabular}{|c|c|c|c|c|}
\hline Menopausal status & & CA125 & Serum HE4 & Urine HE4 \\
\hline \multirow{4}{*}{ Premenopausal } & Malignant & $203.77 \pm 297.39$ & $144.26 \pm 353.39$ & $975.49 \pm 171.86$ \\
\hline & Benign & $140.02 \pm 175.20$ & $64.40 \pm 41.25$ & $788.88 \pm 255.21$ \\
\hline & Controls & $17.45 \pm 7.29$ & $88.81 \pm 62.36$ & $827.38 \pm 260.90$ \\
\hline & $\mathrm{p}$ & 0.016 & 0.5 & 0.078 \\
\hline \multirow{4}{*}{ Postmenopausal } & Malignant & $988.49 \pm 1467.67$ & $247.11 \pm 297$ & $962.79 \pm 271.18$ \\
\hline & Benign & 27.9 & 110.50 & 1200 \\
\hline & Controls & $13.36 \pm 5.20$ & $84.25 \pm 82.83$ & $779 \pm 270.21$ \\
\hline & $p$ & 0.088 & 0.249 & 0.145 \\
\hline
\end{tabular}




\section{DISCUSSION}

Epithelial ovarian cancer (EOC) is the most lethal malignancy of the female genital tract. Its indolent clinical course, together with the absence of early symptoms, are the reasons why patients are diagnosed in advanced stages, with the 5 -year survival of nearly $30 \%$ [13]. Numerous studies have evaluated alternative markers for diagnosing EOC at early stages (stage I-II), in order to increase life expectancy. Our study included 50 patients with an adnexal mass, and 30 controls. Serum CA125 and HE4, urine HE4 and bcl2, and saliva bcl 2 levels were evaluated in these patients, and the diagnostic values of these markers were investigated.

CA125 is the single most commonly used serum marker in EOC [14]. Following the discovery of this antigen, various authors investigated and confirmed its diagnostic and prognostic role in EOC. Unfortunately, CA125 is elevated in only about $50 \%$ of patients with early-stage EOC $[6,15]$. CA125 is a much more sensitive and specific assay when used in postmenopausal women, as benign pathologies in premenopausal women limit is diagnostic accuracy in that group [16]. A number of previously conducted studies have shown that the measurement of serum CA125 alone is not adequate in the screening and diagnosis of EOC. Regardless, it is effective in the follow-up of patients with pre-treatment elevated values $[17,18]$. Therefore, alternative methods for the evaluation of suspicious adnexal masses have been proposed [19]. With the introduction of monoclonal antibody-dependent assays, Human epididymis protein-4 (HE4) has emerged as a sensitive and specific tumor marker, with promising results in EOC [20,21]. It is synthesized in the endoplasmic reticulum and perinuclear Golgi apparatus within the cancer cells. Previous studies have shown that $32 \%$ of EOC patients had elevated HE4 levels in serum [22]. Its sensitivity is comparable to that of CA125, however it has higher specificity for EOC [21]. In a study by Havrileski et al., it was demonstrated that a combined use of HE4 with other tumor markers was effective in the early diagnosis of EOC both, in the primary and recurrent disease settings [23, 24]. Moore et al., evaluated CA125, SMRP, HE4, CA72-4, activin, inhibin, osteopontin, epidermal growth factor receptor and found that the combination of CA125 and HE4 had a sensitivity of $76 \%$ and a specificity of $95 \%$ in differential diagnosis between benign and malignant adnexal masses $[25,26]$.

In our study, three tumor markers (CA125, HE4 and bcl2) were evaluated in sera, saliva and urine. Serum CA125, serum HE4 and urine HE4 levels were significantly higher in patients with ovarian malignancy. Interestingly, urine bcl2 levels were significantly higher in patients without OC. Moreover, saliva bcl 2 levels were similar in benign and malignant cases $(p>0.05)$.

In a study by Huhtinen et al., 129 patients with endometriosis were compared in terms of HE4 with $14 \mathrm{EOC}$, 16 en- dometrial cancer, and 66 control patients [27]. Mean serum HE4 in patients with EOC was $268.3 \pm 2670$ pM, whereas it was $43.5 \pm 13.4 \mathrm{pM}$ in patients with endometriosis. Although mean serum HE4 levels were higher in our study, we also observed similar results. It was concluded that in benign cases, HE4 was as high as in patients with EOC.

The ROMA scores (Risk of Ovarian Malignancy Algorithm) were assessed for our study groups [25]. We found that ROMA was most sensitive for postmenopausal patients with ovarian malignancy. In a study by Montagnana et al., 55 patients with EOC were compared with 49 patients with an adnexal mass and 49 healthy women. Serum CA125, serum HE4 and ROMA scores were evaluated [28]. It was demonstrated that ROMA was useful primarily in postmenopausal women, with very little additional effectiveness in premenopausal patients. HE4 may be used solely in premenopausal patients. Jacobs et al., concluded that the concomitant use of CA125 and HE4 was not a very effective strategy in clinical practice [29]. Our findings also confirmed these results.

Urine tumor markers have been previously assessed in a number of studies, with the assumption that low molecular weight $(<50 \mathrm{kd}$ ) markers could be filtrated from the glomerula and be detected prior to the elevation in serum. In a study by Anderson et al., bcl2, which is an anti-apoptotic protein, was assessed in the urine of patients with EOC, benign adnexal masses, and healthy controls. It was shown that urine bcl 2 was elevated in patients with EOC, and was a useful and non-invasive marker by itself or in combination with other tumor markers [8]. On the contrary, in our study we found that urine bcl 2 was higher in the control group. This finding may be due to a relatively small sample size, and should be further evaluated. Urine HE4 levels were also evaluated in our study. We found that urine HE4 was significantly higher in patients with OC. In a study by Hellstrom et al., 79 patients with EOC (15 early and 64 advanced stage), 20 patients with benign adnexal masses, and 36 healthy subjects were compared in terms of urine HE4. They found the sensitivity of urine HE4 in early EOC to be $86.6 \%$, and $90.5 \%$ in advanced EOC [9]. The sensitivity for detecting EOC was similar for serum and urine HE4. In our study, we also found that serum HE4 levels were significantly higher in malignant cases.

Saliva of the patients has also been previously assessed in the diagnosis of OC. A number of immunologic, metabolic, gastrointestinal and vitamin synthesis disorders, parenchymal and secretory alterations occur within the salivary glands. As saliva is easy to obtain, with minimal risk of infectious disease spread, it is preferred in the diagnosis of several diseases. In a previous study, although serum CA125 was found to be significantly higher in sera of patients with ovarian cancer, there were no differences in terms of saliva 
CA125 levels between EOC and controls [10]. In our study, we only assessed the bcl2 levels in the saliva of the study subjects but we did not detect significant differences in malignant, benign, and control subjects.

Currently, the available markers which are measured in various body fluids are not adequately accurate in the preoperative differential diagnosis of adnexal masses [30]. Pelvic exam, serum CA125 transvaginal ultrasound, or a combination of these modalities are frequently used. However, none of these methods are definitive, and demand further surgical evaluation in high-risk patients. It is critical for high-risk patients to be operated in comprehensive oncology centers, preferably by gynecologic oncologists, as the survival rate is higher in these appropriately managed women [31].

\section{CONCLUSIONS}

Our study detected elevated serum CA125, serum HE4 and urine HE4 levels in patients with ovarian cancer. Also, ROMA was found to be a sensitive method of detecting postmenopausal malignant adnexal masses. On the other hand, the diagnostic significance of saliva and urine bcl2 concentrations was not confirmed. These findings should be assessed in future studies with larger sample sizes in order to reach more definite conclusions.

\section{Conflict of interest}

The study was financially supported by the Başkent University School of Medicine Scientific Fund (Project: KA10/62).

\section{REFERENCES}

1. Sankaranarayanan R, Ferlay J. Worldwide burden of gynaecological cancer: the size of the problem. Best Pract Res Clin Obstet Gynaecol. 2006,20 (2), 207-225.

2. http://globocan.iarc.fr/. Published 2008

3. Heintz AP, Odicino F, Maisonneuve P, [et al.]. Carcinoma of the ovary. FIGO 26th Annual Report on the Results of Treatment in Gynecological Cancer. Int J Gynaecol Obstet. 2006, 95 (Suppl 1), S161-S192.

4. Hellstrom I, Hellstrom KE. SMRP and HE4 as biomarkers for ovarian carcinoma when used alone and in combination with CA125 and/or each other. Adv Exp Med Biol. 2008, 622, 15-21.

5. Kişnişçi A, Gökşin E. Malign Over Tümörleri. In: Kişnişçi A, Gökşin E, Durukan T, [et al.], eds. Temel Kadın Hastalıkları Ve Doğum Bilgisi. Ankara, Güneş Kitabevi 1996, 981-1008.

6. Maggino T, Gadducci A, D'Addario V, [et al.]. Prospective multicenter study on CA 125 in postmenopausal pelvic masses. Gynecol Oncol. 1994, 54 (2), 117-123.

7. Aydınlı K, Kaleli S, Atasü T. Jinekolojik Kanserlerin Erken Tanııı. In: Atasü T, Aydını K, eds. Jinekolojik Onkoloji. İstanbul, Logos Yayıncılık 1999, 133-146.

8. Anderson NS, Bermudez Y, Badgwell D, [et al.]. Urinary levels of $\mathrm{BCl}-2$ are elevated in ovarian cancer patients. Gynecol Oncol. 2009, 112 (1), 60-67.

9. Hellstrom I, Heagerty PJ, Swisher EM, [et al.]. Detection of the HE4 protein in urine as a biomarker for ovarian neoplasms. Cancer Lett. $2010,296(1), 43-48$
10. Tay SK, Chua EK. Correlation of serum, urinary and salivary CA 125 levels in patients with adnexal masses. Ann Acad Med Singapore. 1994, 23 (3), 311-314.

11. Agha-Hosseini F, Mirzaii-Dizgah I, Rahimi A, Seilanian-Toosi M. Correlation of serum and salivary CA125 levels in patients with breast cancer. J Contemp Dent Pract. 2009, 10 (6), E001-E008.

12. Moore RG, MacLaughlan S, Bast RC Jr. Current state of biomarker development for clinical application in epithelial ovarian cancer. Gynecol Oncol. 2010, 116 (2), 240-245.

13. Jacobs I, Davies AP, Bridges J, [et al.]. Prevalence screening for ovarian cancer in postmenopausal women by CA 125 measurement and ultrasonography. BMJ. 1993, 306 (6884), 1030-1034.

14. Berek JS, Knapp RC, Malkasian GD, [et al.]. CA 125 serum levels correlated with second-look operations among ovarian cancer patients. Obstet Gynecol. 1986, 67 (5), 685-689.

15. Bast RC Jr, Klug TL, St John E, [et al.]. A radioimmunoassay using a monoclonal antibody to monitor the course of epithelial ovarian cancer. New Engl J Med. 1983, 309 (15), 883-887.

16. Hempling RE. Tumor markers in epithelial ovarian cancer. Clinical applications. Obstet Gynecol Clin North Am. 1994, 21 (1), 41-61.

17. Einhorn N, Sjovall K, Knapp RC, [et al.]. Prospective evaluation of serum CA 125 levels for early detection of ovarian cancer. Obstet Gynecol. 1992, 80 (1), 14-18.

18. Finkler NJ, Benacerraf B, Lavin PT, Wojciechowski C, Knapp RC. Comparison of serum CA 125, clinical impression, and ultrasound in the preoperative evaluation of ovarian masses. Obstet Gynecol. 1988, 72 (4), 659-664.

19. Moore RG, Brown AK, Miller MC, [et al.]. The use of multiple novel tumor biomarkers for the detection of ovarian carcinoma in patients with a pelvic mass. Gynecol Oncol. 2008, 108 (2), 402-408.

20. Drapkin $\mathrm{R}$, von Horsten $\mathrm{HH}$, Lin $\mathrm{Y}$, [et al.]. Human epididymis protein 4 (HE4) is a secreted glycoprotein that is overexpressed by serous and endometrioid ovarian carcinomas. Cancer Res. 2005, 65 (6), 2162-2169.

21. Hellstrom I, Raycraft J, Hayden-Ledbetter M, [et al.]. The HE4 (WFDC2) protein is a biomarker for ovarian carcinoma. Cancer Res. 2003, 63 (13), 3695-3700.

22. Rosen DG, Wang L, Atkinson JN, [et al.]. Potential markers that complement expression of CA125 in epithelial ovarian cancer. Gynecol Oncol. 2005, 99 (2), 267-277.

23. Havrilesky LJ, Whitehead CM, Rubatt JM, [et al.]. Evaluation of biomarker panels for early stage ovarian cancer detection and monitoring for disease recurrence. Gynecol Oncol. 2008, 110 (3), 374-382.

24. Moore RG, Brown AK, Miller MC, [et al.]. Utility of a novel serum tumor biomarker HE4 in patients with endometrioid adenocarcinoma of the uterus. Gynecol Oncol. 2008, 110 (2), 196-201.

25. Moore RG, McMeekin DS, Brown AK, [et al.]. A novel multiple marker bioassay utilizing HE4 and CA125 for the prediction of ovarian cancer in patients with a pelvic mass. Gynecol Oncol. 2009, 112 (1), 40-46.

26. Moore RG, Jabre-Raughley M, Brown AK, [et al.]. Comparison of a nove multiple marker assay vs the Risk of Malignancy Index for the prediction of epithelial ovarian cancer in patients with a pelvic mass. Am J Obstet Gynecol. 2010, 203 (3), e221-e226.

27. Huhtinen K, Suvitie P, Hiissa J, [et al.]. Serum HE4 concentration differentiates malignant ovarian tumours from ovarian endometriotic cysts. $\mathrm{Br}$ J Cancer. 2009, 100 (8), 1315-1319.

28. Montagnana M, Danese E, Ruzzenente O, [et al.]. The ROMA (Risk of Ovarian Malignancy Algorithm) for estimating the risk of epithelial ovarian cancer in women presenting with pelvic mass: is it really useful? Clin Chem Lab Med. 2011, 49 (3), 521-525.

29. Jacob F, Meier M, Caduff R, [et al.]. No benefit from combining HE4 and CA125 as ovarian tumor markers in a clinical setting. Gynecol Oncol. 2011, 121 (3), 487-491.

30. Partridge EE, Barnes MN. Epithelial ovarian cancer: prevention, diagnosis, and treatment. CA Cancer J Clin. 1999, 49 (5), 297-320.

31. Kehoe S, Powell J, Wilson S, Woodman C. The influence of the operating surgeon's specialisation on patient survival in ovarian carcinoma. $\mathrm{Br}$ J Cancer. 1994, 70 (5), 1014-1017. 